\title{
Research on Transportation Capacity of Urban Rail Transit Network System
}

\author{
Yanyan Liu ${ }^{a}$, Junfang Jia ${ }^{b}$ \\ School of Beijing Jiaotong University, Beijing 100044, China. \\ a17125678@bjtu.edu.com, bjfjia@bjtu.edu.com
}

\begin{abstract}
In the context of the gradual networking of urban rail transit lines, the focus of urban rail transit capacity will shift from the line to the network. At the same time, with the development of computer information technology, virtual cities have become more and more important for the planning construction and management of physical cities. Based on the concept of transportation capacity of urban rail transit network system ,and the analysis of the factors affecting the transportation capacity of urban rail transit network system, firstly the paper proposes the principle of calculating the transportation capacity of each subsystem of urban rail transit network, and then establishes the model of the urban rail transit network system transportation capacity, next analyzes the case and verify the model with the virtual city as the background, indicating that the proposed concept and calculation model have practical significance.
\end{abstract}

Keywords: urban rail transit; network system; transportation capacity.

\section{Introduction}

Despite domestic urban rail transit (Metro + Light Rail) approval is becoming more stringent under the pressure of local government debt risk, there are more than 40 cities building urban railroads, and more than 30 cities in operation since the Beijing Metro has proposed construction and operation. China has ushered in the urban rail era. According to the statistics of China Urban Rail Transit Association, by the end of 2017, the mileage of urban rail transit in China has increased to $5021.7 \mathrm{~km}$, and the annual passenger traffic has reached 18.5 billion passengers (excluding Hong Kong, Macao and Taiwan). In 2017, China's urban rail transit construction investment was 476.2 billion yuan, and the line under construction was 6,246 kilometers.

China's urban rail transit has maintained an orderly development, which has played an important role in improving the quality and efficiency of urban public transport supply, alleviating urban traffic congestion, guiding the optimization of urban spatial structure and improving the urban environment. However, from the perspective of long-term planning, more cities in China will form a relatively complete urban rail transit network, thus bringing the advantages of urban rail transit to the extreme. The urban rail transit network system is composed of subsystems such as stations and lines, and its transportation capacity is not a simple sum of the capacity of the station and the transmission capacity of the line. At the same time, it is only one-sided to study the transportation capacity of each subsystem and the transportation capacity of the network system is only limited by its subsystems. Therefore, the research on the transportation capacity of urban rail transit network system is of great significance to the full utilization of urban rail transit, and it is also the basis of urban rail transit planning and management.

\section{Urban Rail Transit Network System Transport Capacity Definition}

The urban rail transit network is composed of interconnected rail transit lines within the city and the connection between the rail transit lines mainly depends on the lines or transfer stations. The urban rail transit network structure is essentially the distribution of the lines and stations that constitute the network. For example, the network structure that can appear in three rail transit lines has a triangle shape, a Japanese shape, and a tree shape.

The network transportation capacity is an extension of line capacity. Its definition should not only reflect the characteristics of the network's own transportation capacity, but also provide certain metrics for certain transportation capacity indicators. Rail transit provides transport services for 
travellers. Depending on the characteristics of the transport product, transport services are related to displacement, ie the definition of transport capacity of the network should reflect the transport distance. In addition, the extension of the length of the rail transit line, that is, the extended network will undoubtedly increase the transport capacity of the network. It can be seen that the network transport capacity is also related to the network scale. In summary, the urban rail transit network system transport capacity refers to the maximum passenger transport turnover that the system can complete in a unit time under the conditions of the existing rail transit equipment, driving organization method and transportation demand of the urban rail transit network system. The unit is ten thousand persons*kilometers. The concept of the urban rail transit network system transportation capacity mainly consists of the design transportation capacity, the use transportation capacity and the potential transportation capacity of the network system.

(1) the design transportation capacity

The urban rail transit network system design transport capacity is equivalent to the maximum capacity, theoretical capacity or theoretical maximum capacity. It refers to the maximum passenger turnover that can be completed by the urban rail transit network system in a unit time after technical transformation or new line construction. The utilization rate of the network system is $100 \%$. Because the design transportation capacity of the network system is not directly related to the change of transportation demand, it can be used to evaluate the objective results of the extension of the urban rail transit line and the increase of the capacity of the network after the network is expanded without considering the traffic volume. And can be used to indicate the maximum transport capacity that can be possessed in the case of a particular transport organization and equipment [1].

(2) the use transportation capacity

The use transportation capacity of the urban rail transit network system refers to the ability to deduct the basis of the design transportation capacity of the network system, mainly considering the impact of daily random factors on transportation capacity and the coordination of various subsystems. Specifically, under the existing transportation organization mode, fixed equipment and driving organization mode, personnel factors, and specific passenger transportation demand conditions, the passenger traffic volume that can be actually completed in a unit time. In the case of the calculated network system design transport capacity is greater than the existing transport capacity, but the network system transport capacity is very tight, this phenomenon needs to be explained by the concept of the use transportation capacity of network system. In addition, the connection between supply and demand of transportation capacity in time and space distribution can also be reflected by its concept.

(3) the potential transportation capacity

The potential transportation capacity of the network system is mainly based on the design and use transportation capacity. It can be calculated by the difference between the two. It means that the urban rail transit network has been used, but due to some reasons (the transportation capacity of several sections in the line is different, some related lines have not been completed, transportation organization requirements, etc.), there is no ability to use it in the network. This part of the unused capacity is related to the ability to be wasted due to personnel errors, environmental factors and equipment inspection and maintenance, and the ability to reserve as needed [2].

\section{Analysis of Factors Affecting Transportation Capacity of Urban Rail Transit Network System}

(1) analysis of factors affecting station capacity

The station is a place for passenger flow distribution, and it is the most basic point element of the urban rail transit network. The capacity of the station has an important impact on the transportation capacity of the entire network. In some cases, station capacity not only limits the efficiency of passengers arriving at the platform, but also limits the efficiency of the train, thereby reducing the available capacity. The improvement in this area is mainly the responsibility of the transportation provider. It needs to consider the following factors: the capacity limitation of the toll system, the station flow restriction, the station capacity, the lack of space for the station, and the ability of the 
ticketing system. As far as the station passenger flow restriction is concerned, the design of the platform should be based on the long-term passenger flow, so as to meet the passengers' travel demand. In addition, the ability of the ticketing system is also an important factor restricting the transportation capacity. The number and location of the ticket vending machines should be reasonably arranged according to the specific conditions of the station, reducing the passengers' stay time at the station and improving the passengers' travel efficiency.

(2) analysis of factors affecting line capacity

Line capacity refers to the maximum number of trains passing fixed technical equipment of rail transit lines in unit time (usually peak hours) under the constraints of certain types of vehicles, signalrelated equipment, and certain driving organization methods. The number of trains. The station dwell time and minimum tracking interval are the main determinants of line capacity. In the design capability, the minimum tracking interval is closely related to the length of the occlusion zone, the parameters of the signal system, the length of the train, the method of ticket verification and the capacity limitation of the station.

(3) analysis of factors affecting train capacity

The number of passengers per vehicle is a key issue in the calculation of train capacity. It is affected by many factors, such as the number of seats in the train, the length of the platform, and the width of the vehicle. The degree of congestion of a vehicle can generally be used as an indicator for evaluating vehicle capabilities. The system has two main indicators to evaluate vehicle capabilities: one is a design-oriented capability indicator and the other is a general availability capability. If a certain type of vehicle is selected, the calculation of the ability is relatively simple. The factors involved are: seat number, standing area, standing density, standing efficiency, wheelchair adjustment factor, and baggage adjustment factor.

(4) analysis of other factors

In addition to the influence of urban rail transit station capacity, line capacity and train capacity, the passenger transport demand structure and the choice of travel route are also the factors affecting urban rail transit capacity [3]. From the perspective of transportation products, the concept of network transportation capacity also includes the influence factor of network scale. In the case of high driving density, the construction workers' maintenance operations, the form of opening the sunroof and the length of time required for maintenance will lead to changes in the capacity of the line and the method of driving organization. Urban rail transit network system as a big joint motivation, personnel quality, equipment failure and external factors (climate, environmental changes, etc.) will affect rail transit production.

\section{Calculation Model of Transportation Capacity of Urban Rail Transit Network System}

\subsection{Calculation Principle of Transportation Capacity of Urban Rail Transit Network Each Subsystem}

\subsubsection{Station Capacity Analysis}

(1) station distribution capacity calculation

According to the main factors affecting the station capacity, such as the channel ability, the ability of the escalator and stairs, the use ability of Automatic Fare Collection system (AFC), and the capacity of the platform and station hall, etc., the final station capacity is obtained after the capacity calculation of the influencing factors. The calculation method is shown in Fig.1. 


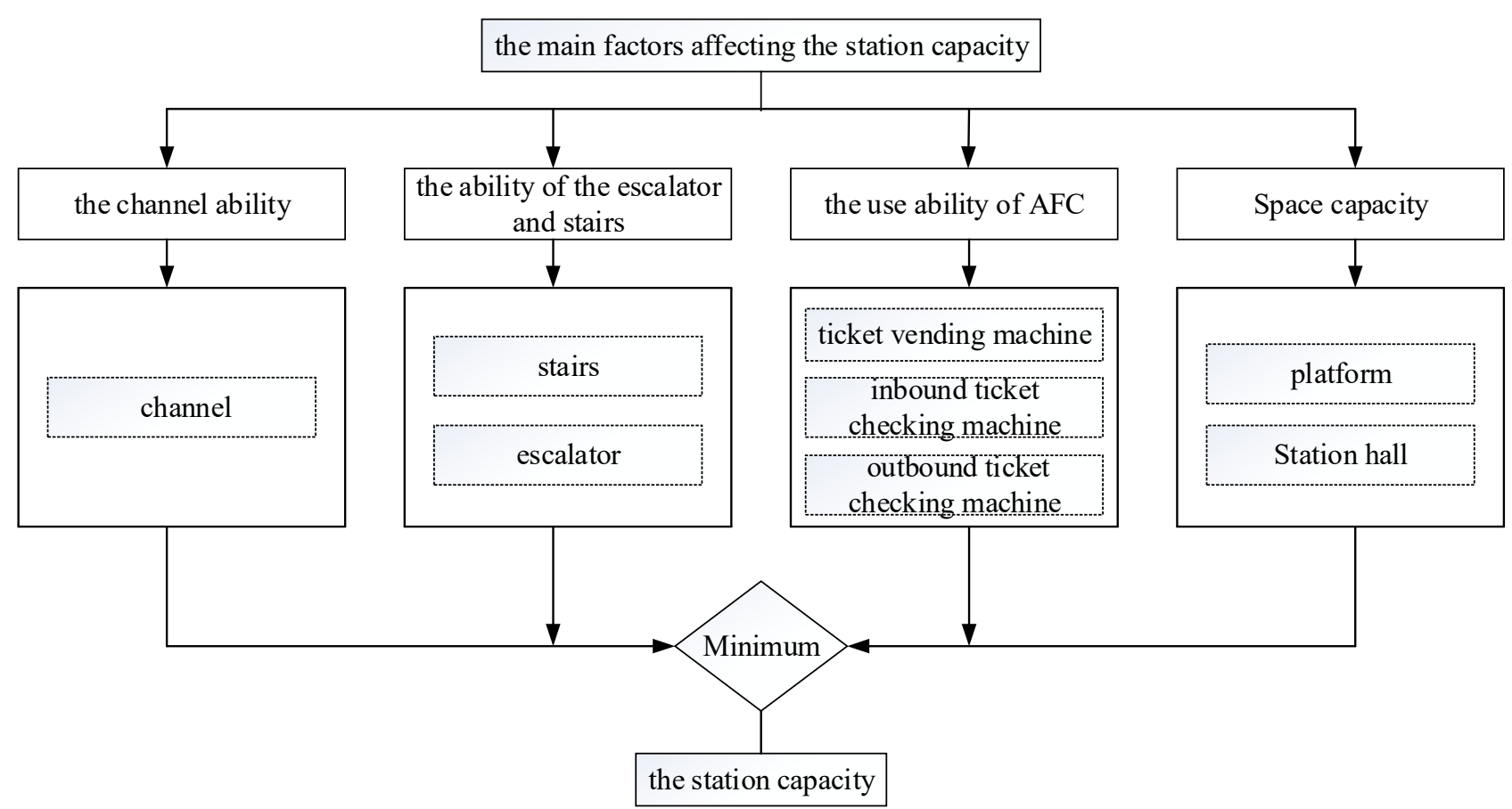

Fig. 1 Calculation methods of station capacity

According to the "cask principle" in economics, the final station capacity depends on the ability of the weakest link in the station equipment, so the following method for calculating the final station capacity is obtained:

$$
N_{\text {station }}=\min \left(N_{\text {chamnel }}, N_{\text {stairs }}, N_{\text {checking }}, N_{\text {vending }}, V_{\text {platform }}, V_{\text {hall }}\right)
$$

In the formula:

$N_{\text {station }}$-station capacity;

$N_{\text {channel }}$-channel capability;

$N_{\text {stairs }}$ - passing capacity of escalators and stairs;

$N_{\text {checking }}$ - ticket checking equipment capacity;

$N_{\text {vending }}$ - ticket vending equipment capacity;

$N_{\text {platform }}$ - platform capacity;

$N_{\text {hall }}$-station hall capacity.

(2) station transfer capacity calculation

The capacity of the urban rail transit transfer system is similar to the concept of station distribution capacity. It refers to the maximum number of transfer passengers of one rail transit line to another during the unit time (usually refers to a day, night, year or peak hour), and it is limited to the use of a certain type of driving organization, based on the distribution of staff responsibilities, the number of existing fixed and active equipment. The final calculation formula for the transfer capacity:

$$
N_{n}^{i-j}=\min \left(N_{\text {stairs }}^{i}, N_{\text {chamel }}, N_{\text {escalator }}, N_{\text {strairs }}, N_{\text {checking }}, N_{\text {stairs }}^{j}\right)
$$

In the formula:

$N_{n}^{i-j}$ the transfer capacity of station $\mathrm{n}$ from line $\mathrm{i}$ to line $\mathrm{j}$;

$N_{\text {stairs }}^{i}$ the capacity of stairs connecting the line i platform;

$N_{\text {stairs }}^{j}$ the capacity of stairs connecting the line j platform;

$N_{\text {channel }}, N_{\text {escalator }}, N_{\text {stairs }}$ the capacity of channels, escalators and stairs in transfer passenger flow services;

$N_{\text {checking }}$ the capacity of the ticket gate during the transfer process. 


\subsubsection{Line Capability Analysis}

Line capacity is an overall reflection of the capacity of the rail transit system, where the size of the traffic density is related to the line ability. Usually it consists of the capacity of tracking the train in the interval, the capacity of the train to turn back at the turn-around station, and the capacity of passing through the intermediate station, where the return capability of the train is often an element limiting the line capacity [4].

The general formula for calculating the final capacity of a line:

$$
n_{\text {final }}=\min \left(n_{\max }, n_{\text {turn-around }}\right)=\min \left(\frac{3600}{t_{\text {interval }}}, \frac{3600}{t_{\text {departure }}}\right)=\frac{3600}{\max \left(t_{\text {interval }}, t_{\text {departure }}\right)}
$$

In the formula:

$n_{\text {final }}$ the maximum number of trains that the line finally passed per hour, column;

$n_{\max }$ the maximum number of trains that the line can pass per hour, column;

$n_{\text {turn-around }}$ the maximum number of trains that the returning equipment can fold back per hour, column;

$t_{\text {interval }} \rightarrow$ minimum train interval, s;

$t_{\text {departure }}$ the minimum departure interval at the turn-back station, s.

\subsubsection{Train Capacity Analysis}

The train capacity is the number of passengers per vehicle multiplied by the number of vehicles per train. Each of the vehicle passengers is affected by a number of factors, which is a key research issue in the capacity calculation. Formula for calculating train capacity:

$$
P_{\text {train }}=m \times p
$$

In the formula:

$$
\begin{aligned}
& P_{\text {train }} \text { - the train capapcity, person; } \\
& m \text { - the number of trains marshalling; } \\
& p \text { - the passerger number of a vehicle, person. }
\end{aligned}
$$

\subsubsection{Available Capability Analysis}

Available capacity refers to the maximum number of passengers that can be transported in a direction of $1 \mathrm{~h}$ in a certain line under the condition that passenger demand is allowed to diverge. It is the product of design capability and peak divergence coefficient. Design capability refers to the amount of passenger space passing through a certain point within a certain direction of a certain line. It is equivalent to the maximum capacity, theoretical ability or theoretical maximum capacity. It is generally difficult to achieve, so it is necessary to define an available capability. The design capability is calculated by multiplying the train capacity by the line capacity [5].

Available capacity calculation formula:

$$
P=n_{\text {final }} \times P_{\text {train }} \times \mathcal{E}
$$

In the formula:

$P$ - available capacity, person;

$\varepsilon$ - the peak divergence coefficient, generally take $0.70-0.95$.

\subsection{Calculation Model of Transportation Capacity of Urban Rail Transit Network System}

With the increase of urban rail transit lines, urban rail transit lines have gradually become networks. When passengers transfer between lines, they will cause some passenger flow to overlap. Therefore, the transportation capacity of the urban rail transit network system is not simply superimposing the point system and line system transportation capacity, but by the line capacity and station capacity joint constraints, while considering the size of the network. 
(1) calculation of the design transportation capacity of network system

The network system design transport capacity is the theoretical maximum capacity, and its calculation method is as follows:

$$
N_{\text {design }}=10^{-4} \sum_{i=1}^{n} \sum_{j=1}^{t}\left(p_{i, j}^{(\text {up })} \times l_{i, j}+p_{i, j}^{(\text {down })} \times l_{i, t-j+1}\right)
$$

In the formula:

$N_{\text {design }}$ - the design transportation capacity of the road network system within an hour, ten thousand persons* kilometers;

$n$ - the number of road network lines, $i=1,2, \ldots, n$;

$t$ - the number of segments of line i divided by each transfer point $j=1,2, \ldots, t$;

$p_{i, j}^{\text {(up) }}$-he maximum transport capacity of the jth segment in the upward direction of line $\mathrm{i}$ within an hour, person;

$l_{i, j}$ - line length of the jth section of the line $\mathrm{i}$ in the upward direction, $\mathrm{km}$;

$p_{i, j}^{\text {(down) }}$ - the maximum transport capacity of the jth segment in the downward direction of line $\mathrm{i}$ within an hour, person.

among them,

$$
\begin{aligned}
& p_{i, j}^{\text {(上) }}=p_{i, j-1}^{\text {(上) }}-p_{i, j-1}^{\text {(上换) }}+p_{j-1, i}^{\text {(换上) }} \\
& p_{i, 1}^{\text {(上) }}=p_{i}=n_{\mathrm{i}, \text { 最终 }} \times p_{i, \text { 列 }} \times \varepsilon
\end{aligned}
$$

In the formula:

$p_{i, j}^{\text {(up) }}$ - the maximum transport capacity of the jth segment in the upward direction of line $\mathrm{i}$ within an hour, person;

$p_{i, j-1}^{\text {(up) }}$ - the maximum transport capacity of the $\mathrm{j}$-1 th segment in the upward direction of line $\mathrm{i}$ within an hour, person;

$p_{i, j-1}^{\text {(uptransfer) }}$ the train arrives in the upward direction of the line $\mathrm{i}$, and the number of passengers who transfer to the other line at the $\mathrm{j}-1$ th transfer point of the line $\mathrm{i}$ within an hour, person; $p_{j-1, i}^{\text {(transferup) }}$ - the train arrives in the other line ,the number of passengers who transfer to the upward direction of the line $\mathrm{i}$ at the $\mathrm{j}-1$ th transfer point of the line $\mathrm{i}$ within an hour,person; $p_{i, 1}^{\text {(up) }}$ - the maximum conveying capacity of the first section of the line $\mathrm{i}$ with an hour, person; $p_{i}$ - the available capacity of line i, person;

$n_{i, f i n a l}$ - the maximum number of trains that line i will eventually pass with an hour,column; $p_{i, \text { train }}$ - the number of passengers that the line i transports, person.

(2) calculation of the use transportation capacity of network system

The calculation method for the transportation capacity of the network system is as follows:

$$
N_{\text {use }}=f\left(\boldsymbol{n}_{1, \text { use }}(T), \cdots, \boldsymbol{n}_{i, \text { use }}(T), \cdots, \boldsymbol{n}_{m, \text { use }}(T) ; \theta\right)
$$


In the formula:

$N_{\text {use }}$ - the use transportation capacity of the road network system in the T period, ten thousand *kilometers;

$f(\bullet)$ - the mutual coordination and coupling relation of the each subsystem capacity;

$n_{i, u s e}(T)$ - the use transportation capacity of subsystem $\mathrm{i}$ in the T period, ten

thousand *kilometers;

$m$-number of subsystems, $i=1,2, \cdots, m$;

$\theta$-random interference factor;

$T$-time period, time unit (one hour, one day, etc.).

The transportation capacity of the network system is variable, because the utilization method of each equipment is changing when the technical requirements are not changed, that is, the calculation of the transportation capacity is a dynamic viewpoint. At the same time, the location of the weak link in the network system can also be changed because of the imbalance of transportation. The balance of each subsystem cannot be considered in isolation and static, and the transportation capacity calculation method of the network system should be considered from the perspective of global and dynamic.

(3) calculation of the potential transportation capacity of network system

In general, the formula for calculating the potential transport capacity of a network system is as follows:

$$
N_{\text {potential }}=N_{\text {design }}-N_{\text {use }}
$$

If the network transportation capacity cannot be fully used due to the inspection and repair of the equipment, the external environment, the staff's mistakes, etc., the calculation formula of the potential transportation capacity of the network system should be expressed as:

$$
N_{\text {potential }}=N_{\text {design }}-N_{\text {use }}-N_{\text {invalid }}
$$

In the formula:

$N_{\text {potential }}$ - the potential transportation capacity of the road network system , ten thousand persons*kilometers;

$N_{\text {invalid }}$ - the invalid transportation capacity of the road network system , ten thousand persons*kilometers.

\section{Case Analysis}

\subsection{Basic Data}

A city plans to build four subway lines in the near future, as shown in Fig.2. Metro Line 1 is eastwest, with a total length of 28.8 kilometers, and is routed to four interchange stations. It is expected that Metro Line 1 will have the largest passenger traffic among the four lines. Metro Line 2 is a subway ring line. Its route is a regular rectangular shape with a total length of 23.5 kilometers. It intersects with Metro Line 1 and Metro Line 5 to form four interchange stations. The length of Metro Line 3 is 24 kilometers. The line runs east-west and then north-south. Metro Line 5 has a total length of 27 kilometers. It is expected to build a 14.8-kilometer underground line and a 12.7-kilometer ground and overhead line. This line is a north-south subway line. 


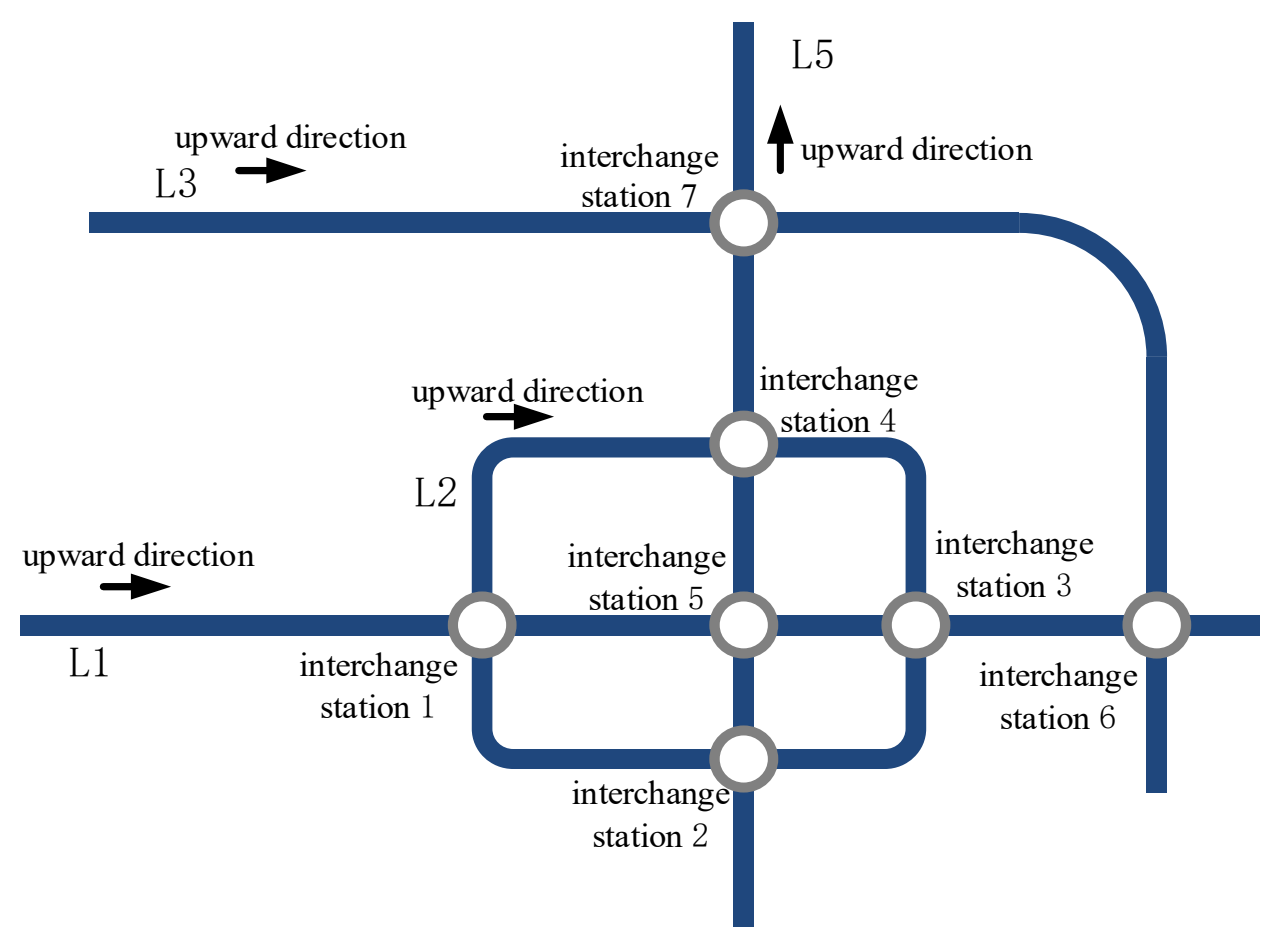

Fig. 2 A recent map of urban rail transit network in a city

It is known that the lengths of the sections of Metro Line 1 are: $l_{1,1}=16 \mathrm{~km}, l_{1,2}=6 \mathrm{~km}, l_{1,3}=2 \mathrm{~km}$, $l_{1,4}=3 \mathrm{~km}, l_{1,5}=1.8 \mathrm{~km}$. The lengths of the sections of Metro Line 2 are: $l_{2,1}=9.5 \mathrm{~km}, l_{2,2}=6 \mathrm{~km}, l_{2,3}=2 \mathrm{~km}$, $l_{2,4}=6 \mathrm{~km}$. The lengths of the sections of Metro Line 3 are: $l_{3,1}=11 \mathrm{~km}, l_{3,2}=10 \mathrm{~km}, l_{3,3}=3 \mathrm{~km}$. The lengths of the sections of Metro Line 5 are: $l_{5,1}=6 \mathrm{~km}, l_{5,2}=1 \mathrm{~km}, l_{5,3}=5 \mathrm{~km}, l_{5,4}=3 \mathrm{~km}, l_{5,5}=12 \mathrm{~km}$. (Note: The length order of each line segment is in the order of the length of each segment in the uplink direction.)

Considering the speed limit of each line, the length of the occlusion zone, the parameters of the signal system, the train return method, the train formation and the staffing, etc., according to the calculation method of the transportation capacity of each subsystem, the design capability of each line is obtained. Then, according to the calculated transfer ability of the seven transfer stations (the transfer ability refers to the maximum number of transfer passengers), the transport capability of each line segment is calculated. The urban rail transit network system transport capacity considers the influence of the network scale. Under the condition that the length of each line section is known, according to the model of the network system design transport capacity, finally the calculated design transportation capacity is 10.56 million persons*kilometers.

\subsection{Case Analysis}

This paper mainly considers the influencing factors of station, line and train capacity when studying the transportation capacity of urban rail transit network system. The space-time characteristics of passenger flow and the overall service level of network have not been considered in the impact of network transportation capacity [6]. Therefore, only the design transportation capacity of the network system is calculated. Referring to the existing urban rail transit network system capacity in major cities, the calculation result of the network system design transport capacity is basically consistent with the actual situation, indicating that the network system design transportation capacity model has guiding significance for the planning and design of urban rail transit network.

In order to fully exploit the potential of the urban rail transit network system, the following measures can be used to improve the transportation capacity of the urban rail transit network system:

(1) Optimize urban rail transit vehicles. On the one hand, choose large capacity vehicle. The urban rail transit lines with large passenger traffic, such as Metro Line 1 in the example, can be used with large vehicles with appropriate number of doors and door width. On the other hand, optimize the interior of the vehicle. The basic starting point of this measure is to increase the vehicle load by changing the double seat to a single seat or changing the vertical fixed seat to a folding seat under 
certain conditions of the vehicle size. This measure also plays an important role in improving the service level of trains, which in turn affects the overall service level of the network.

(2) Increase the marshalling number of trains. This measure can greatly improve the transportation capacity. If 8 sets are used, the driving interval is 3 minutes, and 20 pairs of trains can be actually operated. However, the expansion of trains is constrained by the length of the platform and the economics of operation. At the same time, the large group formation increases the interval and prolongs the waiting time of passengers. This needs to be combined with the passenger's travel needs, considering the spatial and temporal distribution characteristics of the passenger flow.

(3) Optimize the network structure. The rationality of the station and line layout has an important impact on improving the operational efficiency of the urban rail transit network system. In addition, to ensure that the passenger flow can flow well in the entire network, it is necessary to deeply consider the problem of matching transport capacity between lines and stations from the perspective of network connection [7].

\section{Summary}

With the large-scale construction of urban rail transit lines, many urban rail transit constructions in China have developed from single-line plans to network plans. Therefore, deepening the transportation capacity of urban rail transit network systems has become an important issue to be solved in urban rail transit operations. The research on the network system transportation capacity is of great significance for optimizing transportation organization, rationally allocating various resources, and improving the efficiency of urban rail transit. In addition, it is necessary to ensure that the development scale of the urban rail transit network is matched with the actual demand, the construction rhythm and supporting capacity are adapted to achieve standardized, orderly and sustained healthy development.

\section{References}

[1]. YANG Jie: Study on the strategy for the organization of train operation based on the network of rail transit (Master degree, Beijing Jiaotong University, China 2006). p. 16-18.

[2]. ZHANG Yimei: Study on urban rail transit system carrying capacity based on network (Master degree, Beijing Jiaotong University, China 2009). p. 47-48.

[3]. HU Jianqiang: Study on calculating methods of the passenger transport capacity of urban rail transit network (Master degree, Beijing Jiaotong University, China 2006). p. 22-25.

[4]. ZHAN Guobao, LIU Mingshu, XU Ruihua. Analysis on carrying capacity for URT train turnback at way side station[J]. Urban Rail Transit Research. Vol. 8 (2005) No. 6, p. 31-35.

[5]. MAO Baohua. Urban rail transit system operation management. China Communications Press,2006, p.116-120.

[6]. HU Shuai: A simulation-based method for assessing passenger transport capacity on an urban rail network (Master degree, Beijing Jiaotong University, China 2014). p. 18-24.

[7]. FANG Xiaohong, ZHOU Leishan, WANG Yongming. Research on network coordination of urban rail transit. Integrated transportation. (2008) No. 6, p. 63-66. 